\title{
The epidemiology and treatment of conjunctivitis at Urgent Care Centres in Israel
}

This article was published in the following Dove Press journal:

Clinical Ophthalmology

\author{
Ariela Gordon-Shaag (iD) \\ Deena Rachel Zimmerman $\left(\mathbb{D}^{2}\right.$ \\ Einat Shneor (D) ${ }^{\prime}$ \\ 'Department of Optometry, Hadassah \\ Academic College, Jerusalem 910100I, \\ Israel; ${ }^{2}$ TEREM Emergency Medical \\ Centers, Jerusalem 9546303, Israel
}

Purpose: Conjunctivitis is the most common ocular condition diagnosed at emergency departments (ED) in the USA, although it is generally not an emergent condition. Treatment of conjunctivitis at Urgent Care Centres (UCC) could offer lower cost than ED. This study describes the demographics and outcomes of a cohort presenting with conjunctivitis to a nationwide UCC system.

Methods: This retrospective study included 17 branches of UCC. Electronic Medical Record data (November 2015-October 2016) of patients diagnosed with conjunctivitis or ocular disorder were retrieved. Data included gender, age, diagnosis, treatment, discharge status and temperature. Patients without conjunctivitis, presenting to UCC during this period served as the control. Results were compared to all ED patients in Israel (from a public report). Descriptive statistics, Chi-square and Z-proportion test were used.

Results: Altogether, 602,074 patients presented to UCC, of which 5,045 $(0.84 \%, 95 \%$ CI $0.74-0.94 \%$ ) were diagnosed with conjunctivitis. Conjunctivitis was more prevalent among young males $(0-14, p<0.001)$ and older females. The conjunctivitis cohort at UCC was significantly younger than the ED cohort $(p<0.01)$. UCC treated and released home $96.7 \%$ (95\% CI 96.2-97.2\%) of cases. This is significantly higher than the treatment rate for the entire UCC cohort $(93.2 \%$, CI 93.2-93.3\%, $p<0.05)$. Treatment in most cases $(82.0 \%, 95 \%$ CI 80.9-83.0\%) involved the prescription of ocular antibiotic.

Conclusion: Similar to previous studies at ED, conjunctivitis patients are primarily young males. Most patients were treated with antibiotics at UCC and released home. This suggests that UCC may be a good venue for treatment of conjunctivitis.

Keywords: conjunctivitis, urgent care centers, emergency department, public health

\section{Introduction}

Conjunctivitis is the most common ocular condition diagnosed in emergency departments (ED) in the United States, accounting for almost one-third of all eyerelated encounters. ${ }^{1-3}$ In Israel, $0.64 \%$ of all ED visits $(18 \%$ of patients presenting with an ocular problem) are due to a diagnosis of conjunctivitis. ${ }^{4}$

Edwards $^{5}$ found that $71.3 \%$ of the cases seen at an ophthalmologic ED were conjunctivitis and suggested that non-ophthalmologists could have treated $69.0 \%$ of these patients. That study highlights the fact that in most cases, conjunctivitis is not an emergency, ${ }^{3}$ and thus it can be handled in less expensive medical settings than hospital-based ED. ${ }^{6}$

Conjunctivitis patients may present to ED because they need care outside of office hours. In many countries, this is provided primarily by ED, resulting in non-emergent acute conditions being treated at ED. ${ }^{7,8}$ Urgent Care Centres (UCC) were conceived
Correspondence: Einat Shneor Department of Optometry, Hadassah Academic College, 37 Hanevi'im Street, 910100 , Jerusalem, Israel

Tel +97252876 7060

Fax +9726250619

Email eshneor@hac.ac.il 
as a solution to this problem. ${ }^{9}$ Urgent care refers to intermittent health care offered in the community for situations that need immediate attention but are not an emergency. UCC offer walk-in community-based care at lower cost than hospital-based ED, while providing more ancillary services than the traditional practitioner's office. They also have greater temporal convenience than primary care office settings, which have delineated hours and schedules often filled in advance. UCC generally are not equipped to deal with trauma, provide resuscitation or admit patients to a hospital - all reasons for seeking ED care. The UCC health care setting has grown worldwide, ${ }^{9}$ in countries such as England ${ }^{10}$ and the USA, ${ }^{11}$ where the concept of UCC was conceived and developed.

This study describes the demographics of patients presenting with conjunctivitis at community-based UCC. Treatment of conjunctivitis at UCC may be able to reduce ED burden, a notion that has not, to the best of our knowledge, been addressed yet.

\section{Materials and methods}

This was a retrospective study of Electronic Medical Record (EMR) data downloaded anonymously from the UCC database. It was approved by the Hadassah Academic College Ethics Committee and adhered to the tenets of the Declaration of Helsinki. The Ethics Committee waived consent since this was an anonymous retrospective study.

\section{Setting}

The study setting is a nationwide UCC system in Israel which had 17 branches at the time of the study. The four largest branches operate $24 \mathrm{hrs}$ a day, 365 days per year. Smaller branches are open evenings and weekends.

All branches provide physician examinations, and radiological and laboratory services. Staff training includes the assessment of visual acuity (Snellen test), ocular motility, pupillary reactions, lid eversion and fluorescein staining followed by examination with cobalt blue light. The entire system uses an EMR system that manages all elements of the clinical examination.

Each EMR record contains the following demographic data in standard format (chosen from a pulldown menu): clinic code, visit date, gender and age (to two significant figures). Other clinical data are recorded in standard format, such as chief complaint, diagnosis (each EMR may include several diagnoses), treatment(s) given in clinic, prescriptions given at discharge, discharge status (referred to ED or sent home) and temperature. In addition, each EMR record has patient history, physical exam, assessment and treatment plan in free text.

\section{Study population}

All participants were patients of all ages and both genders who presented to UCC between November 1, 2015 and October 31, 2016 (365 days).

The inclusion criteria were a diagnosis of conjunctivitis based on a complete or partial review of the entire file by the investigators (the equivalent of the ICD-9 372.XX codes). Conjunctivitis was diagnosed by UCC physicians as patients presenting with red eye without a history of injury.

Patients who presented to UCC in the past $48 \mathrm{hrs}$ or who had an ocular problem that was not conjunctivitis (eg, foreign body in the eye) were excluded from this study.

The process of identifying patient files that met the inclusion and exclusion criteria for this study are summarized in Figure 1. The diagnosis field of the UCC database was searched with the terms "conjunctivitis" and "eye disorder" (Figure 1A). The records were divided into two excel spreadsheets based on terms in the diagnosis field: "Conjunctivitis" and "To be reviewed" (Figure 1B). "Conjunctivitis" (Figure 1C) included cases with the diagnosis term "conjunctivitis" without the following diagnosis terms "foreign body", "foreign body of eye", "abrasion" or "corneal disorder. "To be reviewed" (Figure 1D) included files with the diagnosis term "eye disorder" as well as files for which the diagnosis terms included "foreign body", "foreign body of eye", "abrasion" or "corneal disorder", with "conjunctivitis". In addition, "To be reviewed" included the diagnosis term "conjunctivitis" with diagnosis terms that suggested corneal and/or conjunctival injury, such as "abrasion" and "animal bite".

Files in "Conjunctivitis" with terms in the chief complaint field that hinted at corneal injury were moved to "To be reviewed" (Figure 1E). This included the following chief complaint terms: "foreign body", "foreign body in the eye", "injury in the eye", "automobile accident", "work accident", "insect bite", "ocular trauma" and "head trauma".

All records in the "To be reviewed" spreadsheet were reviewed by the investigators to determine if they met the inclusion criteria and excluded when appropriate (Figure 1F). 


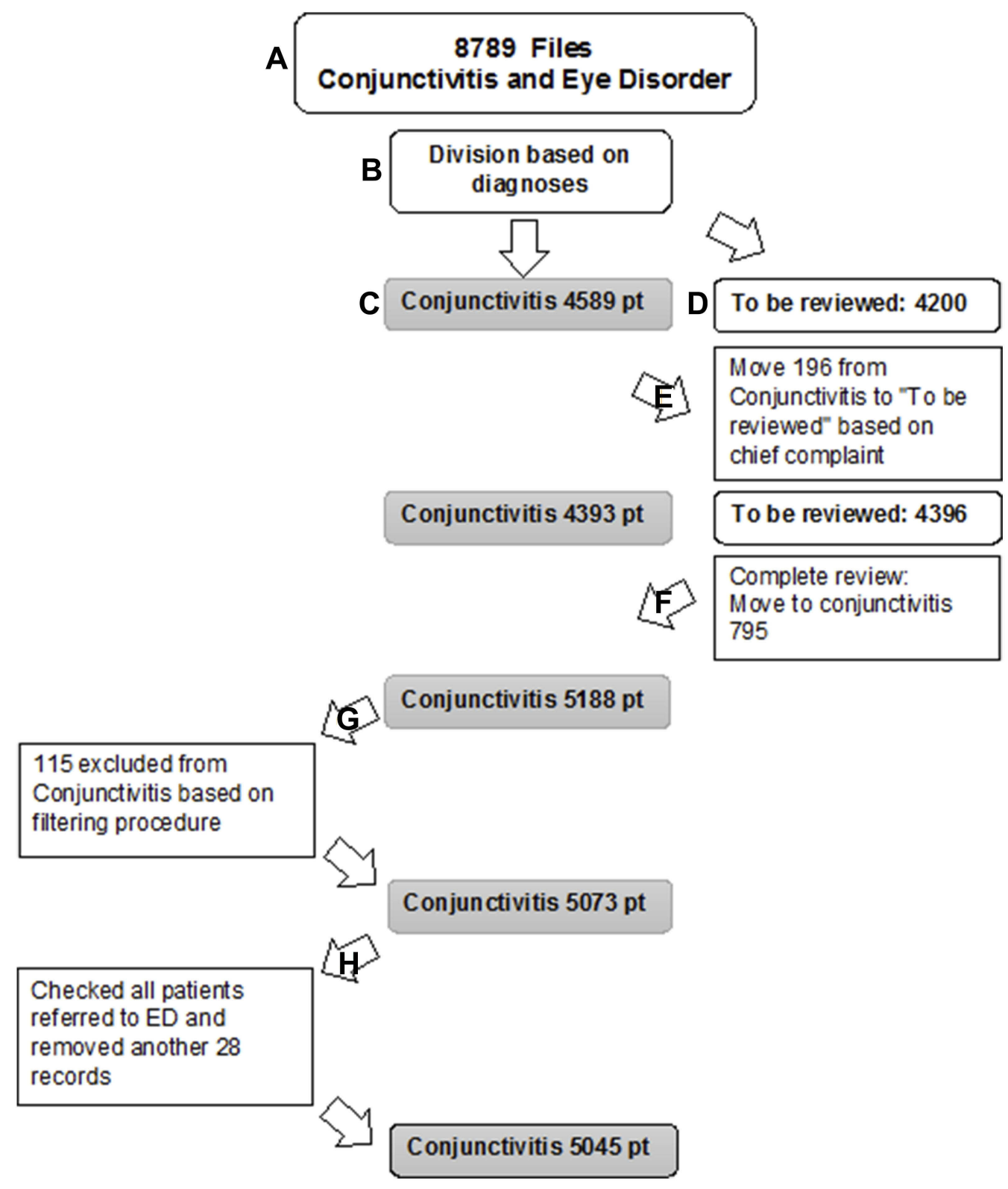

Figure I Flowchart describing the process of identification of patients who met the inclusion/exclusion criteria. Numbers refer to the number of patients at each stage of the process.

The conjunctivitis file was filtered (Figure 1G) to identify cases that met the exclusion criteria. The fields history, physical examination and treatment were searched with the following terms in Hebrew and in English, allowing for various spelling: material, cleaning, welding, spray, penetrate, pepper (for pepper spray) foreign or body, snow (Israeli version of silly string), irrigation, contact lens, finger, wood, work and soap. All files of patients referred to ED were reviewed by experts, as well (Figure 1H).

As a control for conjunctivitis, anonymous data were downloaded from all patients presenting to UCC during the same period, to control for demographic biases.
Data on all patients presenting to ED in Israel in 2015, along with a sub-cohort who were diagnosed with conjunctivitis, were collected from a public report by the State of Israel Ministry of Health Information Division, which included the number of patients per age cohort. ${ }^{4}$

A sampling error for the prevalence of conjunctivitis was calculated for the population size of Israel (8 million) with $95 \% \mathrm{CI}$ and found to be $0.12 \%$.

\section{Data analysis}

Conjunctivitis and all UCC patients were grouped into age cohorts according to the age groups in the report from the 
Table I Age and gender distribution of conjunctivitis cohort

\begin{tabular}{|c|c|c|c|c|c|}
\hline \multirow[t]{2}{*}{ Age group } & \multicolumn{2}{|c|}{ Male } & \multicolumn{2}{|c|}{ Female* } & \\
\hline & $\mathbf{N}$ & $\%$ & $\mathbf{N}$ & $\%$ & \\
\hline 0 & 322 & $57.8 \% *$ & 235 & $42.2 \%$ & $\chi 2=64.08, d f=10, p<0.001$ \\
\hline $1-4$ & 567 & $56.9 \% *$ & 429 & $43.1 \%$ & \\
\hline $5-14$ & 300 & $58.8 \% *$ & 210 & $41.2 \%$ & \\
\hline $15-17$ & 101 & $48.8 \%$ & 106 & $51.2 \%$ & \\
\hline$|8-2|$ & 118 & $43.9 \%$ & $15 \mid$ & $56.1 \% *$ & \\
\hline $22-34$ & 475 & $52.3 \%$ & 433 & $47.7 \%$ & \\
\hline $35-44$ & 329 & $57.8 \% *$ & 240 & $42.2 \%$ & \\
\hline $45-54$ & 161 & $49.5 \%$ & 164 & $50.5 \%$ & \\
\hline $55-64$ & 148 & $41.3 \%$ & 210 & $58.7 \% *$ & \\
\hline $65-74$ & 100 & $45.5 \%$ & 120 & $54.5 \% *$ & \\
\hline$>75$ & 56 & $44.4 \%$ & 70 & $55.6 \% *$ & \\
\hline All & 2677 & $53.1 \%$ & 2368 & $46.9 \%$ & \\
\hline
\end{tabular}

Note: *Significant difference.

Abbreviations: $\chi 2$, Chi-square; $\mathrm{df}$, degrees of freedom.

State of Israel Ministry of Health report. ${ }^{4}$ The patients were grouped according to gender and whether they were referred to ED.

Descriptive statistics were used to describe the characteristics of all observations and of each group of subjects separately according to age and gender. Prevalence and 95\% CI were calculated. The difference between males and females was based on the two-sided Chi-square test. Tests were adjusted for all pairwise comparisons within a row of each innermost sub-table using the bonferroni correction.

To examine proportions between the nominal variables in the study (age of the patient), the Z-proportion test was used. The larger the $Z$-value between the two proportions, the more significant the result, while the sign of $Z$-value indicates the direction. All tests were two-tailed and values of $p<0.05$ were considered statistically significant. Analyses were performed in Microsoft Excel and SPSS version 24.

When testing the cohort according to the day of visit, only UCC branches that are open all year were included in the analysis.

Records with missing data were excluded.

\section{Results}

During the study period, 602,074 patients presented to UCC of which $5,045(0.84 \%, 95 \%$ CI $0.74-0.94 \%)$ were identified as having conjunctivitis. Altogether 3,744 patient files were excluded (Figure 1).

The average age $\pm \mathrm{SD}$ of the conjunctivitis cohort was $25.3 \pm 22.9$ years old (range 0.01-97.78 years). Among conjunctivitis patients, women were significantly older than men $(27.14 \pm 23.52$ vs $23.71 \pm 22.21, p<0.001)$.

Table 1 and Figure 2 demonstrate the distribution by age and gender of patients who arrived to UCC with conjunctivitis. Figure 2 shows a bimodal distribution of patient with a steep peak at ages 5 and younger and another broad peak from 22 to 44 . For children age 14 and younger, significantly more boys present with conjunctivitis $(<14$ years old, Table $1, p<0.001)$. Women have more conjunctivitis at older ages (55 and older, Table 1, $p<0.001)$.

In Table 2, the cohort of patients presenting to UCC with conjunctivitis was compared to two different cohorts as controls: all patients who presented to UCC during that time (UCC) and all patients who presented to ED at a similar time (ED). The results show (Table 2) that in terms of age, the conjunctivitis patients are significantly different than the entire UCC cohort and patients presenting to ED. A significantly larger percentage of the conjunctivitis cohort was younger than five in comparison with the entire UCC cohort ( $31 \%$ vs $18 \%, p<0.01$, Table 2 ). However, the trend is opposite in the 18-21, 22-34, 45-54 and above 65 age groups $(p<0.01)$. When comparing the conjunctivitis cohort to all the patients in Israel who presented to ED from a national database ${ }^{4}$ (Table 2 ), there are a higher percentage of conjunctivitis subjects younger than 18 in the UCC cohort $(45 \%$ vs $23 \%, p<0.05)$. However, the trend is opposite at older ages $18-21$ and above 45 , where conjunctivitis cohort contains a lower percentage of subjects $(p<0.01)$. 


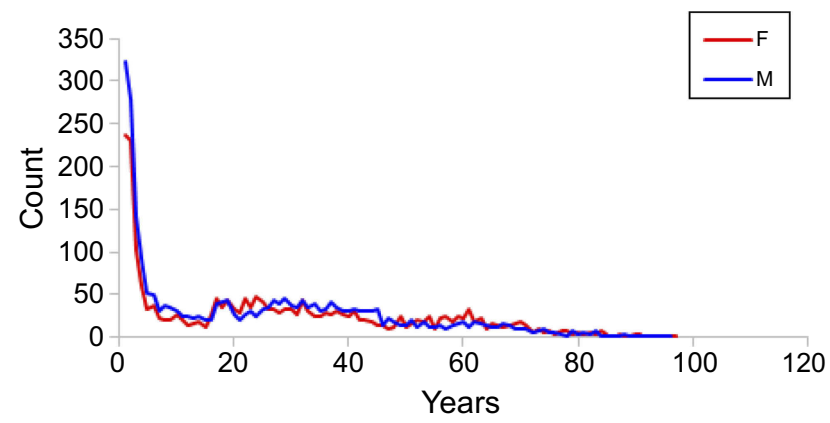

Figure 2 Distribution of conjunctivitis by age and gender. The red and the blue lines represent females and males, respectively.

Table 2 Age distribution in conjunctivitis, UCC and ED cohorts

\begin{tabular}{|c|c|c|c|c|c|c|c|c|}
\hline \multirow[t]{2}{*}{ Age group } & \multicolumn{2}{|c|}{ Conjunctivitis } & \multicolumn{2}{|l|}{ UCC } & \multicolumn{2}{|l|}{ ED } & \multicolumn{2}{|l|}{ Z-test } \\
\hline & $\mathbf{N}$ & $\%$ & $\mathbf{N}$ & $\%$ & $\times 1000$ & $\%$ & $\begin{array}{l}\text { Z-value } \\
\text { (Conjunctivitis-UCC) }\end{array}$ & $\begin{array}{l}\text { Z-value } \\
\text { (Conjunctivitis-ED) }\end{array}$ \\
\hline 0 & 557 & 11.04 & 26,036 & 4.3 & 99 & 3.9 & $23.22 * *$ & $25.98 * *$ \\
\hline $1-4$ & 996 & 19.74 & 81,536 & 13.5 & 187 & 7.3 & $|2.8|^{* *}$ & $33.77^{* *}$ \\
\hline $5-14$ & 510 & 10.11 & 96,555 & 16 & 231 & 9 & $-11.37 * *$ & $2.75^{*}$ \\
\hline $15-17$ & 207 & 4.10 & 25,525 & 4.2 & 77 & 3 & -0.35 & $4.57^{* *}$ \\
\hline$|8-2|$ & 269 & 5.33 & 35,867 & 6 & 190 & 7.4 & $-1.99 *$ & $-5.6 I^{* *}$ \\
\hline $22-34$ & 908 & 18.00 & 116,046 & 19.3 & 467 & 18.2 & $-2.33^{*}$ & -0.37 \\
\hline $35-44$ & 569 & 11.28 & 65,168 & 10.8 & 283 & 11.1 & 1.09 & 0.41 \\
\hline $45-54$ & 325 & 6.44 & 51,338 & 8.5 & 300 & 11.7 & $-5.23 * *$ & $-11.62 * *$ \\
\hline $55-64$ & 358 & 7.10 & 45,339 & 7.5 & 250 & 9.7 & -1.07 & $-6.23 * *$ \\
\hline $65-74$ & 220 & 4.36 & 31,097 & 5.2 & 218 & 8.5 & $-2.7 * *$ & $-10.54 * *$ \\
\hline $75-84$ & 93 & 1.84 & 19,848 & 3.3 & 205 & 8 & $-5.79 * *$ & $-16.12 * *$ \\
\hline$>85$ & 33 & 0.65 & 7,719 & 1.3 & 125 & 4.9 & $-4.07^{* *}$ & $-13.98 * *$ \\
\hline All & 5045 & 100.0 & 602,074 & 100 & 2,563 & 100 & & \\
\hline
\end{tabular}

Note: $p<0.05^{*} p<0.01 * *$.

Abbreviations: UCC, Urgent Care Centres; ED, emergency departments; Z test, Z-proportion test.

The national ED database contained information about patients with conjunctivitis according to age group. During a similar period, 17,101 patients who presented directly to ED were diagnosed with conjunctivitis ( $0.65 \%$ of all ED patients). The age distribution was compared in conjunctivitis patients presenting to UCC and ED (Table 3). The UCC cohort was younger, with higher percentage of patients younger than 18 and a lower percentage 18 and older $(p<0.01)$.

Successful treatment of a condition at UCC can be measured by the referral rate to ED. We found significantly fewer ED referrals of patients with conjunctivitis than from the entire UCC cohort ( $3.33 \%$ vs $6.8 \%, p<0.01)$. This trend was true for all age groups $(p<0.05)$ except for $15-17,22-34,45-54$ and $>85$, where no differences were observed. The patients referred to ED $(\mathrm{N}=168)$ were significantly older than those sent home $(36.85 \pm 22.20$ vs $24.92 \pm 22.82, p<0.0001)$. The most common reason for
ED referral was at least one of the following symptoms: pain, photophobia or reduced vision $(\mathrm{N}=88,52.4 \%, 95 \%$ CI $44.8-59.9 \%$ ). This was followed by patients who had been taking topical antibiotics for conjunctivitis but whose symptoms were getting worse ( $\mathrm{N}=20,11.9 \%, 95 \%$ CI 7.0 $16.8 \%)$, suspected ocular herpes ( $\mathrm{N}=9,5.4 \%, 95 \%$ CI 2.0 $8.8 \%$ ) and patients with a significant ocular history such as recent cataract surgery $(\mathrm{N}=7,4.2 \%, 95 \%$ CI $1.1-7.2 \%)$.

The weekend in Israel is Friday and Saturday, with Friday being a short workday in some industries. Community health clinics typically have limited hours on Friday morning and are closed from Friday noon to Sunday morning. One would expect more UCC visits during the weekend and this is indeed the case (Table 4). However, the conjunctivitis cohort had a significantly higher percentage of patients during the weekend when compared to UCC ( $52 \%$ vs $34 \%, p<0.01)$. 
Table 3 Age distribution of UCC and ED conjunctivitis patients

\begin{tabular}{|l|l|l|l|l|l|}
\hline \multirow{2}{*}{ Age group } & \multicolumn{2}{l|}{ Conjunctivitis UCC } & \multicolumn{2}{l|}{ Conjunctivitis ED } & Z-test \\
\cline { 2 - 6 } & $\mathbf{N}$ & $\%$ & $\mathbf{N}$ & $\%$ & $\begin{array}{l}\text { Z-value } \\
\text { (Conjunctivitis -ED) }\end{array}$ \\
\hline $\mathbf{0 - 4}$ & & & & $40.07^{* *}$ \\
$\mathbf{5 - 1 4}$ & 1,553 & 30.8 & 1,483 & 8.7 & $5.96^{* *}$ \\
$\mathbf{1 5 - 1 7}$ & 510 & 10.1 & 1,291 & 7.5 & $6.45^{* *}$ \\
$\mathbf{1 8 - 2 |}$ & 207 & 4.1 & 417 & 2.4 & $-18.13^{* *}$ \\
$\mathbf{2 2 - 4 4}$ & 269 & 5.3 & 2,569 & 15.0 & $-4.42^{* *}$ \\
$\mathbf{4 5 - 6 4}$ & 1,477 & 29.3 & 5580 & 32.6 & $-10.87^{* *}$ \\
$\mathbf{6 5 - 7 4}$ & 683 & 13.5 & 3,472 & 20.3 & $-8.50^{* *}$ \\
$\mathbf{7 7 5}$ & 220 & 4.4 & 1,344 & 7.9 & $-8.75^{* *}$ \\
Total & 126 & 2.5 & 933 & 5.5 & 100.0 \\
\hline
\end{tabular}

Note: $p<0.05^{*} p<0.01 * *$.

Abbreviations: UCC, Urgent Care Centres; ED, emergency departments; Z test, Z-proportion test.

Table 4 Patients presenting with conjunctivitis and total UCC by day of the week

\begin{tabular}{|l|l|l|l|l|l|}
\hline \multirow{2}{*}{ Day of the week } & \multicolumn{3}{l|}{ Conjunctivitis } & UCC & Z-test (Conjunctivitis -UCC) \\
\cline { 2 - 6 } & $\mathbf{n}$ & $\%$ & $\mathbf{n}$ & $\%$ & Z-value \\
\hline Sun & 474 & 9.7 & 88,796 & $14.1 \%$ & $-8.8 I^{* *}$ \\
Monday & 510 & 10.5 & 83,479 & $13.2 \%$ & $-5.55^{* *}$ \\
Tuesday & 421 & 8.6 & 78,156 & $12.4 \%$ & $-8.03^{* *}$ \\
Wednesday & 501 & 10.3 & 82,906 & $13.1 \%$ & $-5.78^{* *}$ \\
Thursday & 424 & 8.7 & 80,473 & $12.7 \%$ & $-8.37^{* *}$ \\
Friday & 984 & 20.2 & 99,710 & $15.8 \%$ & $8.39 * *$ \\
Saturday & 1567 & 32.1 & 118,413 & $18.7 \%$ & $23.86^{* *}$ \\
Total & $488 \mathrm{I}$ & 100.0 & $\mathbf{6 0 2 , 0 7 3}$ & $100.0 \%$ & $-8.8 I^{* *}$ \\
\hline
\end{tabular}

Note: $p<0.05 * p<0.01 * *$

Abbreviations: UCC, Urgent Care Centres; ED, emergency departments; Z test, Z-proportion test.

Distribution of conjunctivitis among different branches of UCC showed the highest prevalence of conjunctivitis in Eilat, the southernmost city of Israel, which is located in the desert ( $4 \%$ vs $1.5 \%, Z=14.45, p<0.01$ ).

Conjunctivitis can present alone or as part of a systematic adenoviral infection involving fever and other symptoms. Fever of $\geq 38.0^{\circ} \mathrm{C}$ was detected in $7.6 \%$ (95\% CI 6.9-8.3\%) of patients presenting with conjunctivitis. Most of the patients with fever $(94.8 \%, 95 \%$ CI $92.6-97.0 \%)$ were between 1 and 15 years of age. For the subjects without fever $(\mathrm{n}=4661)$, most had a single diagnosis (conjunctivitis or eye disorder) with only $18.4 \%$ (95\% CI $17.3-19.5 \%)$ having multiple diagnoses. In contrast, significantly more subjects with conjunctivitis presenting with fever $(79.4 \%$, 95\% CI 75.4-83.5\%, $p<0.001$ ) had an additional diagnosis such as pneumonia or upper respiratory infection.

We assessed whether a prescription was given to the patients presenting with conjunctivitis. Most conjunctivitis patients $(81.9 \%, 95 \% \mathrm{CI} 80.9-83.0 \%)$ received a prescription for topical antibiotics. For young pediatric patients aged $0-5$ (who comprised $31 \%$ [95\% CI 29.5-32.1\%] of the total cohort), $88.9 \%$ (95\% CI $87.4-90.5 \%$ ) received topical antibiotics. Of these, $78.0 \%$ (95\% CI 75.8-80.2\%) suffered from purulent ocular discharge.

\section{Discussion}

While previous research has described the epidemiology of conjunctivitis in hospital-based $\mathrm{ED},{ }^{12}$ this is the first time this issue has been addressed at a community-based UCC. This study demonstrates that $0.8 \%$ of patients in Israel presenting to UCC are diagnosed with conjunctivitis. These patients are significantly younger than patients in UCC or ED, the majority are male and arrive on the weekend. The overwhelming majority $(96.7 \%)$ were treated at UCC and released home. Most (82\%) of the patient received a prescription for topical antibiotics, while few (7.6\%) had fever. 
There are differences between ED and UCC conjunctivitis patients, particularly the age distribution, with UCC having a much younger cohort than ED. The age distribution at UCC in Israel resembles that found in ED in the USA $^{12}$ and Canada ${ }^{13}$ with a bimodal distribution and peak with young children. The USA study ${ }^{12}$ speculates that the peak in young children is caused by first-time inexperienced parents who bring their children to the ED for non-emergent conditions, such as conjunctivitis. The age distribution of conjunctivitis in Israeli ED suggest a different phenomenon. Perhaps parents of young children are less likely to want to expose their children to ED and thus use the UCC preferentially. Moreover, parents might want immediate treatment for their children, so their children can attend day care. Use of UCC for conjunctivitis is significantly higher on the weekends, supporting this notion. However, an interview-based study would be needed to know if this is the case.

There are significantly more young boys than girls presenting with conjunctivitis, as found in previous studies. ${ }^{12,13}$ The reason for this is not clear.

The conjunctivitis patients treated at UCC may have otherwise presented to the ED, increasing both ED burden and patient cost. ${ }^{3}$ The relative cost of ED to UCC depends on the health care system in question. In Israel, ED costs about three times as much as UCC, ${ }^{9}$ while in the USA, ED is approximately four times more expensive. ${ }^{3,14,15}$ In both cases, there is a considerable savings when patients are treated at UCC. Even in countries with universal national health coverage such as England and Israel, where the patient does not have to pay out of pocket, the use of UCC will result in saving the healthcare system money.

Only $3.3 \%$ of conjunctivitis patients were referred to ED. Most of these referred patients (79\%) did not have an emergent condition; rather they presented with symptoms such as pain, photophobia and blurred vision, which together with conjunctivitis requires an examination by ophthalmologist in the near future. ${ }^{16}$ However, in many countries that offer universal health care, access to ophthalmology is limited. For example, in Israel, all citizens are required by law to belong to one of the country's four health care providers, who allow appointments with ophthalmologists in the community without the requirement of a referral from a primary care physician, for a nominal fee. However, actual patient access to ophthalmologists is limited, while optometrists do not have therapeutic pharmaceutical privileges. Therefore, UCC referred these patients directly to ED, which have an ophthalmologist on call.
The majority of patients $(82 \%)$ received a prescription for topical antibiotics. This finding is similar to previous studies. In the Netherlands, $80 \%$ of patients with conjunctivitis under the care of general practitioners received topical antibiotics. ${ }^{17}$ In a survey carried out in Great Britain, 95\% of general care practitioners report prescribing topical antibiotics for conjunctivitis. ${ }^{18}$ A study in the USA found that $68 \%$ of patients diagnosed with conjunctivitis at UCC filled their prescription for topical antibiotics, ${ }^{19}$ although the actual prescription rate is likely to be higher. ${ }^{20}$

The high rate of antibiotic prescription for conjunctivitis may suggest overuse, since most conjunctivitis is viral. However, in this study, $31 \%$ of the cohort presenting at UCC with conjunctivitis are younger than 6 years of age. Since bacterial pathogens are the most common cause of acute pediatric conjunctivitis, ${ }^{21,22}$ and $78 \%$ of these children had purulent ocular discharge, they are most likely to have suffered from bacterial conjunctivitis. While bacterial conjunctivitis will resolve by itself, antimicrobials can be prescribed to shorten the duration of illness. ${ }^{23}$ Furthermore, many child care providers exclude children with conjunctivitis until antibiotic treatment is provided and this has been shown to impact the decision to prescribe antibiotics. ${ }^{24}$ Moreover, it is difficult clinically to differentiate viral from bacterial conjunctivitis, so providers tend to "err on the side of safety" and prescribe antibiotics "just in case". 20

The high number of cases found in a desert condition is interesting. This may be due to the association between desert dust and conjunctivitis. ${ }^{25}$

The finding that most of the patients did not have fever and when fever was present, it was usually in the presence of another condition, further emphasizes that conjunctivitis is not a systemic disease and does not need to be referred to ED.

The main limitation of the paper is that the medical records do not specifically distinguish between viral, bacterial and allergic conjunctivitis. This is likely to have led to the overuse of antibiotics, which can have negative consequences. However, it is difficult to clinically differentiate bacterial from viral conjunctivitis. ${ }^{20}$ For example, in Great Britain, only $36 \%$ of general care physicians believed that they could discriminate between bacterial and viral infection. ${ }^{18}$

Another limitation is that patients were not followedup after release from UCC. Patients who were sent home might not have improved and went to a primary care physician and/or ophthalmologist for further treatment. However, these patients are not adding to ED burden. 


\section{Conclusion}

$\mathrm{UCC}$ is a good and cheaper alternative to ED for the care of conjunctivitis. It should be encouraged as the first place of examination in locations that have access to UCC. Antibiotics may be over-prescribed, but not more so than at other health care settings. As the system of UCC in Israel was modeled on that in the United States, and the causes of conjunctivitis are similar in most developed countries, it is reasonable to assume these finding can be extrapolated to other countries as well.

\section{Abbreviation list}

UCC, Urgent Care Centres; ED, emergency departments; EMR, Electronic Medical Record; $\chi 2$, Chi- square; df, degrees of freedom; $Z$ test, Z-proportion test; pt, patients.

\section{Acknowledgments}

We would like to thank Zoey Gottleib for her assistance in extracting the data. We would like to thank Bracha Adler, Liraz Israeli, Eti Vaalani and Maria Gogonsku for help in gathering data.

\section{Disclosure}

This research did not receive any specific grant from funding agencies in the public, commercial or not-for-profit sectors. DRZ is an employee of TEREM in the capacity of the Director of Research and a Medical Director but has no proprietary interests or conflicts of interest related to this submission. None of the following authors have any proprietary interests or conflicts of interest related to this submission: AGS and ES.

\section{References}

1. Alabbasi OM, Al-Barry M, Albasri RF, et al. Patterns of ophthalmic emergencies presenting to a referral hospital in Medina City, Saudi Arabia. Saudi J Ophthalmol. 2017;31(4):243-246. doi:10.1016/j. sjopt.2016.03.001

2. Carvalho Rde S, Jose NK. Ophthalmology emergency room at the University of Sao Paulo General Hospital: a tertiary hospital providing primary and secondary level care. Clinics (Sao Paulo). 2007;62 (3):301-308. doi:10.1590/S1807-59322007000300015

3. Channa R, Zafar SN, Canner JK, Haring RS, Schneider EB, Friedman DS. Epidemiology of eye-related emergency department visits. JAMA Ophthalmol. 2016;134(3):312-319. doi:10.1001/ jamaophthalmol.2015.5778

4. Haklai Z, Meron J, Shlichkov G, Appelbaum Y, Aburbeh M. Emergency room visits summary 2015. Health Inf Div. 2015. Available from: https:// www.health.gov.il/PublicationsFiles/emergency_2015.pdf,2017
5. Edwards RS. Ophthalmic emergencies in a district general hospital casualty department. $\mathrm{Br} J$ Ophthalmol. 1987;71(12):938-942. doi:10.1136/bjo.71.12.938

6. Filleul L, Pages F, Wan GC, Brottet E, Vilain P. Costs of conjunctivitis outbreak, Reunion Island, France. Emerg Infect Dis. 2018;24 (1):168-170. doi:10.3201/eid2408.171312

7. O'Keeffe C, Mason S, Jacques R, Nicholl J. Characterising nonurgent users of the emergency department (ED): a retrospective analysis of routine ED data. PLoS One. 2018;13(2):e0192855. doi:10.1371/journal.pone.0192855

8. Morris T, Mason SM, Moulton C, O'Keeffe C. Calculating the proportion of avoidable attendances at UK emergency departments: analysis of the Royal College of Emergency Medicine's Sentinel Site Survey data. Emerg Med J. 2018;35(2):114-119. doi:10.1136/emermed-2017-206846

9. Zimmerman DR. Community-based urgent care in Israel and worldwide. Isr J Health Policy Res. 2013;2(1):38. doi:10.1186/2045-4015-2-38

10. The Royal College of Emergency medicine and The Patients Association. Time to act - urgent care and A\&E: the patient perspective. 2015. Available from: https://www.rcem.ac.uk/docs/Policy/CEM8480Time $\% 20$ to $\% 20$ Act $\% 20$ Urgent $\% 20$ Care $\% 20$ and $\% 20 \mathrm{~A}+\mathrm{E} \% 20$ the $\%$ 20patient\%20perspective.pdf. Accessed December 24, 2018.

11. Yee T, Lechner AE, Boukus ER. The surge in urgent care centers: emergency department alternative or costly convenience? Res Brief. 2013;(26):1-6.

12. Ramirez DA, Porco TC, Lietman TM, Keenan JD. Epidemiology of conjunctivitis in US emergency departments. JAMA Ophthalmol. 2017;135(10):1119-1121. doi:10.1001/jamaophthalmol.2017.3319

13. Szyszkowicz M, Kousha T, Castner J. Air pollution and emergency department visits for conjunctivitis: a case-crossover study. Int J Occup Med Environ Health. 2016;29(3):381-393. doi:10.13075/ ijomeh.1896.00442

14. Mehrotra A, Liu H, Adams JL, et al. Comparing costs and quality of care at retail clinics with that of other medical settings for 3 common illnesses. Ann Intern Med. 2009;151(5):321-328. doi:10.7326/00034819-151-5-200909010-00006

15. Thygeson M, Van Vorst KA, Maciosek MV, Solberg L. Use and costs of care in retail clinics versus traditional care sites. Health Aff (Millwood). 2008;27(5):1283-1292. doi:10.1377/hlthaff.27.5.1283

16. Azari AA, Barney NP. Conjunctivitis: a systematic review of diagnosis and treatment. JAMA. 2013;310(16):1721-1729. doi:10.1001/ jama.2013.280318

17. Rietveld RP, Ter Riet G, Bindels PJ, Schellevis FG, van Weert HC. Do general practitioners adhere to the guideline on infectious conjunctivitis? Results of the second Dutch National Survey of general practice. BMC Fam Pract. 2007;8:54. doi:10.1186/1471-2296-8-54

18. Everitt H, Little P. How do GPs diagnose and manage acute infective conjunctivitis? A GP survey. Fam Pract. 2002;19(6):658-660. doi:10.1093/fampra/19.6.658

19. Shekhawat NS, Shtein RM, Blachley TS, Stein JD. Antibiotic prescription fills for acute conjunctivitis among enrollees in a large United States managed care network. Ophthalmology. 2017;124 (8):1099-1107. doi:10.1016/j.ophtha.2017.04.034

20. Keen M, Thompson M. Treatment of acute conjunctivitis in the United States and evidence of antibiotic overuse: isolated issue or a systematic problem? Ophthalmology. 2017;124(8):1096-1098. doi:10.1016/j.ophtha.2017.05.029

21. Isenberg SJ, Apt L, Valenton M, et al. A controlled trial of povidoneiodine to treat infectious conjunctivitis in children. Am J Ophthalmol. 2002;134(5):681-688. doi:10.1016/S0002-9394(02)01701-4

22. Patel PB, Diaz MC, Bennett JE, Attia MW. Clinical features of bacterial conjunctivitis in children. Acad Emerg Med. 2007;14(1):15. doi:10.1197/j.aem.2006.07.032

23. Richards A, Guzman-Cottrill JA. Conjunctivitis. Pediatr Rev. 2010;31(5):196-208. doi:10.1542/pir.31-5-196 
24. Finnikin S, Jolly K. Nursery sickness policies and their influence on prescribing for conjunctivitis: audit and questionnaire survey. Br J Gen Pract. 2016;66(650):e674-679. doi:10.3399/ bjgp16X686125
25. Chien LC, Lien YJ, Yang CH, Yu HL. Acute increase of children's conjunctivitis clinic visits by Asian dust storms exposure - a spatiotemporal study in Taipei, Taiwan. PLoS One. 2014;9 (10):e109175. doi:10.1371/journal.pone.0109175

\section{Publish your work in this journal}

Clinical Ophthalmology is an international, peer-reviewed journal covering all subspecialties within ophthalmology. Key topics include: Optometry; Visual science; Pharmacology and drug therapy in eye diseases; Basic Sciences; Primary and Secondary eye care; Patient Safety and Quality of Care Improvements. This journal is indexed on PubMed
Central and CAS, and is the official journal of The Society of Clinical Ophthalmology (SCO). The manuscript management system is completely online and includes a very quick and fair peer-review system, which is all easy to use. Visit http://www.dovepress.com/ testimonials.php to read real quotes from published authors.

Submit your manuscript here: https://www.dovepress.com/clinical-ophthalmology-journal 